\title{
Corroborating the equivalence between the Duffin-Kemmer-Petiau and the Klein-Gordon and Proca equations
}

\author{
L. B. Castro* \\ Departamento de Física CFM, Universidade Federal de Santa Catarina (UFSC), 88.040-900 CP. 476, Florianópolis, Santa Catarina, Brazil \\ and Departamento de Física, Universidade Federal do Maranhão (UFMA), Campus Universitário do Bacanga, 65085-580, São Luís, \\ Maranhão, Brazil \\ A. S. de Castro ${ }^{\dagger}$ \\ Departamento de Física e Química, Universidade Estadual Paulista, 12516-410 Guaratinguetá, São Paulo, Brazil
}

(Received 16 January 2014; published 5 August 2014)

\begin{abstract}
It is shown that the Hamiltonian version of the Duffin-Kemmer-Petiau theory with electromagnetic coupling brings about a source term at the current. It is also shown that such a source term disappears from the scenario if one uses the correct physical form for the Duffin-Kemmer-Petiau field, regardless of the choice for representing the Duffin-Kemmer-Petiau matrices. This result is used to fix the ambiguity in the electromagnetic coupling in the Duffin-Kemmer-Petiau theory. Moreover, some widespread misconceptions about the Hermiticity in the Duffin-Kemmer-Petiau theory are discussed.
\end{abstract}

DOI: 10.1103/PhysRevA.90.022101

PACS number(s): 03.65.Pm, 03.65.Ca, 03.65.Ge

\section{INTRODUCTION}

The first-order Duffin-Kemmer-Petiau (DKP) formalism [1,2] describes spin-0 and spin-1 particles and enjoys a richness of couplings not capable of being expressed in the Klein-Gordon (KG) and Proca theories $[3,4]$. The way for introducing the minimal coupling has been a subject of quite some debate. One can introduce the minimal coupling either at the equation of motion or at the Hamiltonian form of the DKP theory, and these ways seem not to be equivalent as already noted by Kemmer in his original work [2]. The main issue is that when the minimally coupled covariant form of the DKP equation is written in Hamiltonian form, there appears an additional term which is called the anomalous term and additionally the energy-momentum tensor is not conserved. Ghose [5] suggested that one should introduce the minimal coupling at the Hamiltonian form of the DKP theory for avoiding the appearance of the anomalous term, and a conserved energy-momentum tensor appears as a bonus. Nowakowski [6] and Lunardi et al. [7] showed that such an anomalous term disappears when the physical components of the DKP field are selected. Struyve et al. [8] analyzed the ambiguity of introducing the minimal coupling and suggested that despite the nonconservation of the energy-momentum tensor, we should introduce the minimal coupling via the covariant form of the DKP equation, in order to obtain the minimally coupled KG theory. Therefore, there exists a discrepancy in how to introduce the minimal coupling and it seems that this discrepancy has still not found a definitive conclusion.

The main purpose of the present paper is to clarify the ambiguity of the electromagnetic coupling in the DKP theory. To achieve this, the continuity equation for a charged boson minimally coupled to the electromagnetic field is analyzed by using both the equation of motion and its Hamiltonian

\footnotetext{
*1rb.castro@ufma.br

†castro@pq.cnpq.br
}

version. It is shown that the charge quadricurrent $J^{\mu}$ has a source term when one uses the Hamiltonian version of the DKP theory. By using a proper set of operators [9] whose algebraic properties make our conclusions independent of the choice for representing the DKP matrices, it is also shown that such a source term disappears from the DKP theory if one uses the correct physical components of the DKP spinor as prescribed in [7]. Therefore, it does not matter if one either put the electromagnetic coupling straight in the Hamiltonian or in the equation of motion, because the current is conserved in both versions of the DKP theory. In addition, some widespread misconceptions about the Hermiticity in the DKP theory diffused in the literature are discussed.

\section{DUFFIN-KEMMER-PETIAU EQUATION}

The DKP equation for a free charged boson is given by [2] (with units in which $\hbar=c=1$ )

$$
\left(i \beta^{\mu} \partial_{\mu}-m\right) \psi=0,
$$

where the matrices $\beta^{\mu}$ satisfy the algebra $\beta^{\mu} \beta^{\nu} \beta^{\lambda}+$ $\beta^{\lambda} \beta^{v} \beta^{\mu}=g^{\mu v} \beta^{\lambda}+g^{\lambda v} \beta^{\mu}$ and the metric tensor is $g^{\mu \nu}=$ $\operatorname{diag}(1,-1,-1,-1)$. That algebra generates a set of 126 independent matrices whose irreducible representations are a trivial representation, a five-dimensional representation describing the spin- 0 particles, and a ten-dimensional representation associated to spin-1 particles [10]. The DKP spinor has an excess of components and the theory has to be supplemented by an equation which allows one to eliminate the redundant components. That constraint equation is obtained by multiplying the DKP equation by $1-\beta^{0} \beta^{0}$, namely

$$
i \beta^{j} \beta^{0} \beta^{0} \partial_{j} \psi=m\left(1-\beta^{0} \beta^{0}\right) \psi, \quad j \text { runs from } 1 \text { to } 3 \text {. }
$$

This constraint equation expresses three (four) components of the spinor by the other two (six) components and their space derivatives in the scalar (vector) sector so that the superfluous components disappear and there only remain the physical components of the DKP theory. The second-order KG and Proca equations are obtained when one selects the spin-0 and 
spin-1 sectors of the DKP theory. The DKP theory has also its Hamiltonian version in the form (see, e.g., [6,7])

$$
i \partial_{0} \psi=H \psi, \quad H=i\left[\beta^{j}, \beta^{0}\right] \partial_{j}+m \beta^{0} .
$$

Note that in this context we can show that $H^{\dagger}=H$. A well-known conserved four-current is given by

$$
J^{\mu}=\frac{1}{2} \bar{\psi} \beta^{\mu} \psi
$$

where the adjoint spinor $\bar{\psi}$ is given by $\bar{\psi}=\psi^{\dagger} \eta^{0}$ with $\eta^{0}=2 \beta^{0} \beta^{0}-1$ in such a way that $\left(\eta^{0} \beta^{\mu}\right)^{\dagger}=\eta^{0} \beta^{\mu}$ (the matrices $\beta^{\mu}$ are Hermitian with respect to $\left.\eta^{0}\right)$. Despite the similarity to the Dirac equation, the DKP equation involves singular matrices, the time component of $J^{\mu}$ given by (4) is not positive definite, and the case of massless bosons cannot be obtained by a limiting process [11]. Nevertheless, the matrices $\beta^{\mu}$ plus the unit operator generate a ring consistent with integer-spin algebra and $J^{0}$ may be interpreted as a charge density. The factor $1 / 2$ multiplying $\bar{\psi} \beta^{\mu} \psi$, of no importance regarding the conservation law, is in order to hand over a charge density conformable to that one used in the KG theory and its nonrelativistic limit [12].

\section{INTERACTIONS IN THE DUFFIN-KEMMER-PETIAU EQUATION}

With the introduction of interactions, the DKP equation can be written as

$$
\left(i \beta^{\mu} \partial_{\mu}-m-U\right) \psi=0,
$$

where the more general potential matrix $U$ is written in terms of 25 (100) linearly independent matrices pertinent to five (ten)dimensional irreducible representation associated to the scalar (vector) sector. In the presence of interaction, $J^{\mu}$ satisfies the equation

$$
\partial_{\mu} J^{\mu}+\frac{i}{2} \bar{\psi}\left(U-\eta^{0} U^{\dagger} \eta^{0}\right) \psi=0 .
$$

Thus, if $U$ is Hermitian with respect to $\eta^{0}$ then four-current will be conserved. The potential matrix $U$ can be written in terms of well-defined Lorentz structures. For the spin-0 (scalar sector) there are two scalar, two vector, and two tensor terms [3], whereas for the spin-1 (vector sector) there are two scalar, two vector, a pseudoscalar, two pseudovector, and eight tensor terms [4]. The tensor terms have been avoided in applications because they furnish noncausal effects $[3,4]$. The condition (6) has been used to point out a misleading treatment in the recent literature regarding analytical solutions for nonminimal vector interactions [13-15].

\section{A. Duffin-Kemmer-Petiau equation with minimal electromagnetic coupling}

Considering only the minimal vector interaction, the DKP equation for a charged boson with minimal electromagnetic coupling is given by

$$
\left(i \beta^{\mu} D_{\mu}-m\right) \psi=0,
$$

where the covariant derivative is given by $D_{\mu}=\partial_{\mu}+i e A_{\mu}$. In this case, the constraint equation (2) becomes

$$
i \beta^{k} \beta^{0} \beta^{0} \partial_{k} \psi-e \beta^{k} \beta^{0} \beta^{0} A_{k} \psi=m\left(1-\beta^{0} \beta^{0}\right) \psi,
$$

and the four-current $J^{\mu}$ retains its form as (4). The DKP theory with minimal electromagnetic coupling has also its Hamiltonian version in the form (see, e.g., $[6,7]$ )

$$
\begin{aligned}
i \partial_{0} \psi= & H \psi, \\
H= & i\left[\beta^{j}, \beta^{0}\right] D_{j}+e A_{0}+m \beta^{0} \\
& +\frac{i e}{2 m} F_{\mu \nu}\left(\beta^{\mu} \beta^{0} \beta^{\nu}+\beta^{\mu} g^{0 \nu}\right),
\end{aligned}
$$

with the electromagnetic field tensor given by $F_{\mu \nu}=\partial_{\mu} A_{\nu}-$ $\partial_{\nu} A_{\mu}$. The last term in $H$ is called the anomalous term because it has no equivalent in the spin-1/2 Dirac theory. For this reason it has been suggested to put the electromagnetic coupling straight in the Hamiltonian instead of the equation of motion [5]. However, it has been shown in Refs. [6,7] that such an anomalous term disappears when the physical components of the DKP field are selected. Since

$$
\left(i F_{0 j} \beta^{j} \beta^{0} \beta^{0}\right)^{\dagger}=-\left(i F_{0 j} \beta^{j} \beta^{0} \beta^{0}\right)+i F_{0 j} \beta^{j} .
$$

$H$ is not equal to $H^{\dagger}$ [16], in opposition to what was adverted in [6]. Because of this, the Lewis-Riesenfeld invariant method for studying time-dependent fields is not straightforwardly applicable as done in Ref. [17], and already criticized in [18].

\section{B. Hamilton form and Hermiticity}

At this level, it is worthwhile to note that the Hamiltonian given by (9) should be Hermitian with respect to $\eta^{0}$ and not with respect a $\beta^{0}$ as was stated by Zeleny [19]. Zeleny argued that an operator, and in particular the Hamiltonian, should be neo-Hermitian $\left(\beta^{0} \hat{O}=\hat{O}^{\dagger} \beta^{0}\right)$. Furthermore, Zeleny claimed that the free Hamiltonian as well as the minimally coupled Hamiltonian are not neo-Hermitian. Nevertheless, it can be easily shown that both of them are Hermitian with respect to $\eta^{0}$, and therefore $\langle H\rangle$ is a real quantity.

\section{Free case}

The Hamiltonian form of the free DKP equation is given by

$$
i \partial_{0} \psi=H \psi, \quad H=i\left[\beta^{j}, \beta^{0}\right] \partial_{j}+m \beta^{0} .
$$

with the constraint equation

$$
i \beta^{k} \beta^{0} \beta^{0} \partial_{k} \psi=m\left(1-\beta^{0} \beta^{0}\right) \psi
$$

From the algebra of matrices $\beta^{\mu}$, it is shown that

$$
\left[\beta^{i}, \beta^{0}\right]^{\dagger}=\left[\beta^{i}, \beta^{0}\right] .
$$

Furthermore $\hat{p}_{\mu}=i \partial_{\mu}$ is Hermitian and commutes with the matrices $\beta^{\mu}$. With all this, we can show that $H^{\dagger}=H$. On the other hand, multiplying (11) by $\beta^{0}$ from the left and using the constraint (12), we obtain

$$
\beta^{0} H=-i \beta^{k} \partial_{k}+m .
$$

Hermitian conjugation of (14) gives

$$
\left(\beta^{0} H\right)^{\dagger}=i \beta^{k} \partial_{k}+m \neq \beta^{0} H
$$


From this result Zeleny [19] concluded that not even the free Hamiltonian form is neo-Hermitian; for this reason he tried to build a neo-Hermitian Hamiltonian. On the other hand, with the correct criteria it can be shown that

$$
\eta^{0}\left(\beta^{0} H\right)=-i \eta^{0} \beta^{k} \partial_{k}+\eta^{0} m,
$$

and applying the Hermitian conjugation on (16) we have that

$$
\left[\eta^{0}\left(\beta^{0} H\right)\right]^{\dagger}=i \beta^{k} \eta^{0} \partial_{k}+\eta^{0} m=\eta^{0}\left(\beta^{0} H\right) .
$$

Therefore, the Hamiltonian form of the free DKP theory is Hermitian with respect to $\eta^{0}$.

\section{Electromagnetic case}

The Hamiltonian form for the minimally coupled case has the form

$$
\begin{aligned}
i \partial_{0} \psi= & H \psi, \\
H= & i\left[\beta^{j}, \beta^{0}\right] D_{j}+e A_{0}^{(1)}+m \beta^{0} \\
& +\frac{i e}{2 m} F_{\mu \nu}\left(\beta^{\mu} \beta^{0} \beta^{\nu}+\beta^{\mu} \eta^{0 \nu}\right) .
\end{aligned}
$$

Furthermore, the constraint becomes

$$
i \beta^{k} \beta^{0} \beta^{0} \partial_{k} \psi-e \beta^{k} \beta^{0} \beta^{0} A_{k} \psi=m\left(1-\beta^{0} \beta^{0}\right) \psi .
$$

Since

$$
\left(i F_{0 j} \beta^{j} \beta^{0} \beta^{0}\right)^{\dagger}=-\left(i F_{0 j} \beta^{j} \beta^{0} \beta^{0}\right)+i F_{0 j} \beta^{j},
$$

$H$ is not equal to $H^{\dagger}$. On the other hand, multiplying (18) by $\beta^{0}$ from the left and using the constraint (19), we obtain

$$
\beta^{0} H=-i \beta^{k} \partial_{k}+e \beta^{k} A_{k}+e \beta^{0} A_{0}+m .
$$

Taking the Hermitian conjugation of (21) we have

$$
\left(\beta^{0} H\right)^{\dagger}=i \beta^{k} \partial_{k}-e \beta^{k} A_{k}+e \beta^{0} A_{0}+m \neq \beta^{0} H,
$$

similarly to the free case. On the other hand, it can be shown that

$$
\eta^{0}\left(\beta^{0} H\right)=-i \eta^{0} \beta^{k} \partial_{k}+e \eta^{0} \beta^{k} A_{k}+e \beta^{0} A_{0}+\eta^{0} m
$$

and, applying the Hermitian conjugation in (23), we obtain

$$
\begin{aligned}
{\left[\eta^{0}\left(\beta^{0} H\right)\right]^{\dagger} } & =i \beta^{k} \eta^{0} \partial_{k}-e \beta^{k} \eta^{0} A_{k}+e \beta^{0} A_{0}+\eta^{0} m \\
& =\eta^{0}\left(\beta^{0} H\right) .
\end{aligned}
$$

Therefore, the Hamiltonian form with electromagnetic interaction of the DKP theory is Hermitian with respect to $\eta^{0}$. Therefore, we can conclude that the operator $H$ of the Hamiltonian form with electromagnetic interaction is neither Hermitian in the standard sense (for the sake of the anomalous term) nor with respect to $\beta^{0}$. It does not matter; $H$ should be Hermitian with respect to $\eta^{0}$ in order to provide real eigenvalues.

\section{FOUR-CURRENT CONSERVED}

Returning to the ambiguity with the electromagnetic coupling, let us begin with the equation of motion. The conservation law for $J^{\mu}$ follows from the standard procedure of multiplying (7) and its complex conjugate by $\bar{\psi}$ from the left and by $\eta^{0} \psi$ from the right, respectively. On the other hand, by carrying through calculations similar to those using the DKP equation, the Schrödinger-like equation (9) leads to

$$
\begin{aligned}
\partial_{\mu} J^{\mu}= & {\left[\left(D_{j}\right)^{*} \bar{\psi}\right] \beta^{0} \beta^{0} \beta^{j} \psi+\bar{\psi} \beta^{j} \beta^{0} \beta^{0} D_{j} \psi } \\
= & \left(\partial_{j} \bar{\psi}\right) \beta^{0} \beta^{0} \beta^{j} \psi+\bar{\psi} \beta^{j} \beta^{0} \beta^{0}\left(\partial_{j} \psi\right) \\
& +i e A_{j} \bar{\psi}\left[\beta^{j}, \beta^{0} \beta^{0}\right] \psi .
\end{aligned}
$$

In this case, one sees that the malediction of a source term falls on $J^{\mu}$.

Up to this point the physical components of the DKP spinor have not come into the story at all. The contradictory results involving the source terms can be solved by following the prescription put forward in Refs. [6,7]. Instead of working with a specific representation for the matrices $\beta^{\mu}$ we choose an alternative way.

\section{A. Scalar sector}

To select the physical component of the DKP field for the scalar sector (spin-0 sector), we define the operator [20]

$$
P=-\left(\beta^{0}\right)^{2}\left(\beta^{1}\right)^{2}\left(\beta^{2}\right)^{2}\left(\beta^{3}\right)^{2},
$$

which satisfies $P^{2}=P, P^{\mu}=P \beta^{\mu}$, and ${ }^{\nu} P=\left(P^{\nu}\right)^{\dagger}=\beta^{\nu} P$. As it is shown in [20], $P \psi$ and $P^{\mu} \psi$ transform as a (pseudo)scalar and a (pseudo)vector under an infinitesimal Lorentz transformation, respectively.

The spin-0 sector can be expressed by the set of operators $\left\{P,{ }^{\mu} P, P^{\mu},{ }^{\mu} P^{\nu}\right\}$ with the properties [9]

$$
\begin{aligned}
P\left(P^{\mu}\right) & =P^{\mu}, \quad\left({ }^{\mu} P\right) P={ }^{\mu} P, \\
\left(P^{\mu}\right) P & =P\left({ }^{\mu} P\right)=0, \\
\left({ }^{\mu} P\right)\left(P^{v}\right) & ={ }^{\mu} P^{\nu}, \quad\left(P^{\mu}\right)\left({ }^{v} P\right)=g^{\mu \nu} P .
\end{aligned}
$$

Hence

$$
\begin{aligned}
P\left({ }^{\mu} P^{v}\right) & =\left({ }^{\mu} P^{v}\right) P=0, \quad\left(P^{\mu}\right)\left(P^{v}\right)=\left({ }^{v} P\right)\left({ }^{\mu} P\right)=0, \\
\beta^{\mu} & =P^{\mu}+{ }^{\mu} P, \quad \bar{\psi} P=(P \psi)^{\dagger},
\end{aligned}
$$

in such a way that the DKP equation becomes

$$
D_{\mu}\left(P^{\mu} \psi\right)=-i m(P \psi), \quad D^{\mu}(P \psi)=-i m\left(P^{\mu} \psi\right),
$$

which provides

$\left(D^{\mu} D_{\mu}+m^{2}\right)(P \psi)=0, \quad\left(D^{\mu} D_{\mu}+m^{2}\right)\left(P^{v} \psi\right)=0$.

These results tell us that all elements of the column matrices $P \psi$ and $P^{\mu} \psi$ obey the KG equation with minimal coupling and that $P^{\mu} \psi$ is expressed in terms of the covariant derivative of $P \psi$. Then, acting $P$ upon the spinor DKP $\psi$ selects the scalar sector of DKP theory, making explicitly clear that it describes a spin-0 particle embedded in a electromagnetic field. Following this innovative view of the DKP spinor, Ref. [7] shows that the redundant components of $\psi$ are projected out, $\psi$ and $P \psi$ are both compatible with gauge invariance, and the anomalous term in the Hamiltonian version has no physical consequence. Now, we return our attention to 
the DKP current. The $P$ algebra implies that

$$
\begin{aligned}
J^{\mu}= & \frac{1}{2} \bar{\psi}\left(P^{\mu}+{ }^{\mu} P\right) \psi \\
= & \frac{i}{2 m}\left\{(P \psi)^{\dagger}\left[D^{\mu}(P \psi)\right]-\left[\left(D^{\mu}\right)^{*}(P \psi)^{\dagger}\right](P \psi)\right\} \\
= & \frac{i}{2 m}\left\{(P \psi)^{\dagger}\left[\partial^{\mu}(P \psi)\right]-\left[\partial^{\mu}(P \psi)^{\dagger}\right](P \psi)\right\} \\
& -\frac{e}{m} A^{\mu}(P \psi)^{\dagger}(P \psi) .
\end{aligned}
$$

This is nothing but the KG current. In other words, the DKP current is equivalent to the KG current. Hence $\partial_{\mu} J^{\mu}=0$, as derived from the DKP equation, can be seen as a natural result. Indeed, it follows from the $P$ algebra that

$$
\beta^{0} \beta^{0} \beta^{j}=P^{j}, \quad \beta^{j} \beta^{0} \beta^{0}={ }^{j} P .
$$

Thus (25) can be written as

$$
\begin{aligned}
\partial_{\mu} J^{\mu} & =\left[\left(D_{j}\right)^{*} \bar{\psi}\right] P^{j} \psi+\left[\bar{\psi}\left({ }^{j} P\right)\right] D_{j} \psi \\
& =\frac{i}{m}\left\{\left[\left(D_{j}\right)^{*}(P \psi)^{\dagger}\right] D^{j}(P \psi)-\left[\left(D^{j}\right)^{*}(P \psi)^{\dagger}\right] D_{j}(P \psi)\right\} \\
& =0
\end{aligned}
$$

\section{B. Vectorial sector}

Now we discuss the vector sector (spin-1 sector) of the DKP theory. Similar to the scalar sector, we can select the physical components of the DKP field for the spin-1 sector, so we define the operator [20]

$$
R^{\mu}=\left(\beta^{1}\right)^{2}\left(\beta^{2}\right)^{2}\left(\beta^{3}\right)^{2}\left[\beta^{\mu} \beta^{0}-g^{\mu 0}\right],
$$

which satisfies $R^{\mu \nu}=R^{\mu} \beta^{\nu}$ and $R^{\mu \nu}=-R^{v \mu}$. As it is shown in [20], $R^{\mu} \psi$ and $R^{\mu \nu} \psi$ transform as (pseudo)vector and (pseudo)tensor quantities under an infinitesimal Lorentz transformation, respectively.

The spin-1 sector can be expressed by the set of operators $\left\{{ }^{\mu} V^{\nu},{ }^{\mu} V^{\nu \lambda},{ }^{\nu \lambda} V^{\mu},{ }^{\nu \lambda} V^{\mu \sigma}\right\}$ [9], with

$$
\begin{aligned}
{ }^{\mu} V^{\nu} & =\left({ }^{\mu} R\right)\left(R^{\nu}\right), \quad{ }^{\mu} V^{\nu \lambda}=\left({ }^{\mu} R\right)\left(R^{\nu \lambda}\right), \\
{ }^{\nu \lambda} V^{\mu} & =\left({ }^{\nu \lambda} R\right)\left(R^{\mu}\right), \quad{ }^{\nu \lambda} V^{\mu \sigma}=\left({ }^{\nu \lambda} R\right)\left(R^{\mu \sigma}\right),
\end{aligned}
$$

where

$$
\begin{aligned}
\left(R^{\mu}\right)\left({ }^{\nu} R\right) & =\left(R^{0}\right) g^{\mu \nu}, \quad\left(R^{\mu}\right)\left(R^{\nu \lambda}\right)=\left(R^{\nu \lambda}\right) g^{\mu 0} \\
\left(R^{\mu}\right)\left(R^{\nu}\right) & =\left(R^{\nu}\right) g^{\mu 0}, \\
\left(R^{\mu \nu}\right)\left({ }^{\lambda} R\right) & =\left(R^{\mu}\right)\left({ }^{\nu \lambda} R\right)=\left(R^{\mu \nu}\right)\left(R^{\lambda}\right)=0 \\
\left(R^{\mu \nu}\right)\left({ }^{\lambda \sigma} R\right) & =\left(R^{0}\right) \Delta^{\mu \nu \lambda \sigma}, \quad \Delta^{\mu \nu \lambda \sigma}=g^{\mu \sigma} g^{\nu \lambda}-g^{\mu \lambda} g^{\nu \sigma} .
\end{aligned}
$$

In view of (36) one has

$$
\begin{aligned}
\left({ }^{\mu} V^{\nu \lambda}\right)\left({ }^{\rho \sigma} V^{\tau}\right) & =\left({ }^{\mu} V^{\tau}\right) \Delta^{\nu \lambda \rho \sigma}, \\
\left({ }^{\mu} V^{\nu \lambda}\right)\left({ }^{\tau} V^{\rho \sigma}\right) & =\left({ }^{\nu \lambda} V^{\mu}\right)\left({ }^{\rho \sigma} V^{\tau}\right)=0, \\
\beta^{\mu} & =\sum_{\lambda}\left({ }^{\mu \lambda} V^{\lambda}+{ }^{\lambda} V^{\lambda \mu}\right), \\
\bar{\psi}\left(R^{0}\right) & =\left(R^{0} \psi\right)^{\dagger} \eta^{00}, \quad \bar{\psi}\left({ }^{i 0} R\right)=\left(R^{i 0} \psi\right)^{\dagger} \eta^{00},
\end{aligned}
$$

in such a way that the DKP equation becomes

$$
\begin{aligned}
D_{\mu}\left(R^{v \mu} \psi\right) & =-i m\left(R^{v} \psi\right), \quad\left(R^{\mu \nu} \psi\right)=-\frac{i}{m} U^{\mu \nu}, \\
U^{\mu \nu} & =D^{\mu}\left(R^{v} \psi\right)-D^{\nu}\left(R^{\mu} \psi\right),
\end{aligned}
$$

which leads to

$$
\begin{aligned}
D_{\mu} U^{\mu \nu}+m^{2}\left(R^{\nu} \psi\right) & =0, \\
D_{\mu}\left(R^{\mu} \psi\right) & =\frac{i e}{2 m^{2}} F_{\mu \nu} U^{\mu \nu} .
\end{aligned}
$$

These results tell us that all elements of the column matrix $R^{\mu} \psi$ obey the Proca equation interacting minimally with an electromagnetic field. So, similar to the scalar sector, this procedure selects the vector sector of DKP theory, making explicitly clear that it describes a spin-1 particle embedded in an electromagnetic field. A little calculation shows that

$$
\begin{aligned}
J^{\mu} & =\frac{1}{2} \sum_{\lambda} \bar{\psi}\left({ }^{\mu \lambda} V^{\lambda}+{ }^{\lambda} V^{\lambda \mu}\right) \psi \\
& =\frac{1}{2} \sum_{\lambda} \bar{\psi}\left({ }^{\lambda} R\right)\left(R^{\lambda \mu} \psi\right)+\frac{1}{2} \sum_{\lambda} \bar{\psi}\left({ }^{\mu \lambda} R\right)\left(R^{\lambda} \psi\right) \\
& =-\frac{i}{2 m} \sum_{\lambda}\left[\left(R^{\lambda} \psi\right)\left(U^{\mu \lambda}\right)^{*}-\bar{\psi}\left({ }^{\lambda} R\right) U^{\mu \lambda}\right],
\end{aligned}
$$

which shows that $J^{\mu}$ is completely equivalent to the Proca current. Moreover, in order to evaluate $\partial_{\mu} J^{\mu}$ we use the relations

$$
\begin{aligned}
& \beta^{0} \beta^{0} \beta^{i}=\sum_{\lambda}\left({ }^{\lambda} R\right)\left(R^{\lambda i}\right)-\left({ }^{0} R\right)\left(R^{0 i}\right)-\left({ }^{0 i} R\right)\left(R^{0}\right), \\
& \beta^{i} \beta^{0} \beta^{0}=\sum_{\lambda}\left(R^{i \lambda}\right)\left(R^{\lambda}\right)-\left({ }^{i 0} R\right)\left(R^{0}\right)-\left({ }^{i} R\right)\left(R^{i 0}\right),
\end{aligned}
$$

so that $(25)$ results in

$$
\begin{aligned}
\partial_{\mu} J^{\mu} & =\bar{\psi}\left(R^{0}\right) D_{i}\left(R^{0 i} \psi\right)+\left[D_{i}^{*} \bar{\psi}\left({ }^{i 0} R\right)\right]\left(R^{0} \psi\right) \\
& =-\left(R^{0} \psi\right)^{\dagger} D_{i}\left(R^{0 i} \psi\right)-\left[D_{i}^{*}\left(R^{i 0} \psi\right)^{\dagger}\right]\left(R^{0} \psi\right) \\
& =0 .
\end{aligned}
$$

Again, the correct physical components of the DKP spinor make $J^{\mu}$ conserved if one uses either the equation of motion or the Hamiltonian. Therefore, there is no problem in introducing the minimal coupling in the equation of motion or in the form Hamiltonian, because these two ways provide a conserved four-current. Recently, the projectors $P, P^{\mu}, R^{\mu}$, and $R^{\mu \nu}$ have satisfactorily been used to find analytical solutions for spin-0 and spin-1 particles $[21,22]$.

\section{FINAL REMARKS}

In summary, using the conservation of the four-current and the correct interpretation of the physical components of the DKP spinor, we tried to clarify the ambiguity of the electromagnetic coupling in the DKP theory. From this point of view, the ambiguity seen by Kemmer in his original work [2] does not exist, because the current is conserved in both versions of the DKP theory if one uses the correct physical components 
of the DKP spinor as prescribed in [7]. Therefore, it does not matter if one either put the electromagnetic coupling straight in the Hamiltonian or in the equation of motion. Furthermore, $J^{\mu}$ reduces to the KG current or to the Proca current when one selects the appropriate sector of the theory, as should be expected from equivalent theories. Thanks to the algebraic properties of the projectors developed in [9] neither representation for the DKP matrices was used for reaching this conclusion, even if the physical fields depend on the explicit representation of the DKP matrices. Also, we analyzed the Hermiticity of the Hamiltonian and we showed that the operator $H$ of the Hamiltonian form is neither Hermitian in the usual sense nor with respect to $\beta^{0}$ as argued by Zeleny [19]. As a matter of fact, the operator $H$ should be Hermitian with respect to $\eta^{0},\left[\eta^{0}\left(\beta^{0} H\right)\right]^{\dagger}=\eta^{0}\left(\beta^{0} H\right)$, in order to provide real eigenvalues. Finally, our results corroborate and complement the results presented in [6,7] and also they shed some light on some widespread misconceptions about the Hermiticity of the Hamiltonian form in the DKP theory.

\section{ACKNOWLEDGMENTS}

The authors are indebted to the anonymous referee for an excellent and constructive review. This work was supported in part by means of funds provided by CAPES and CNPq. This work was partially done during a visit (L.B.C.) to UNESP, Campus de Guaratinguetá.
[1] G. Petiau, Acad. R. Belg., A. Sci. Mém. Collect. 16, 2 (1936); N. Kemmer, Proc. R. Soc. A 166, 127 (1938); R. J. Duffin, Phys. Rev. 54, 1114 (1938).

[2] N. Kemmer, Proc. R. Soc. A 173, 91 (1939).

[3] R. F. Guertin and T. L. Wilson, Phys. Rev. D 15, 1518 (1977).

[4] B. Vijayalakshmi, M. Seetharaman, and P. M. Mathews, J. Phys. A 12, 665 (1979).

[5] P. Ghose, D. Home, and M. N. S. Roy, Phys. Lett. A 183, 267 (1993); P. Ghose, ibid. 191, 362 (1994); Found. Phys. 26, 1441 (1996).

[6] M. Nowakowski, Phys. Lett. A 244, 329 (1998).

[7] J. T. Lunardi, B. M. Pimentel, R. G. Teixeira, and J. S. Valverde, Phys. Lett. A 268, 165 (2000).

[8] W. Struyve, W. De Baere, J. De Neve, and S. De Weirdt, Phys. Lett. A 322, 84 (2004).

[9] E. Fischbach, M. M. Nieto, and C. K. Scott, J. Math. Phys. 14, 1760 (1973).

[10] A. I. Akhiezer and V. B. Berestetskii, Quantum Electrodynamics (John Wiley \& Sons, New York, 1965).

[11] R. A. Krajcik and M. M. Nieto, Phys. Rev. D 10, 4049 (1974).
[12] T. R. Cardoso, L. B. Castro, and A. S. de Castro, J. Phys. A: Math. Theor. 43, 055306 (2010).

[13] T. R. Cardoso, L. B. Castro, and A. S. de Castro, Can. J. Phys. 87, 857 (2009); 87, 1185 (2009).

[14] T. R. Cardoso, L. B. Castro, and A. S. de Castro, J. Phys. A: Math. Theor. 45, 075302 (2012).

[15] L. B. Castro and L. P. de Oliveira, AdHEP 2014, 784072 (2014).

[16] T. R. Cardoso, L. B. Castro, and A. S. de Castro, Phys. Lett. A 372, 5964 (2008).

[17] M. Merad and S. Bensaid, J. Math. Phys. 48, 073515 (2007).

[18] L. B. Castro and A. S. de Castro, J. Math. Phys. 51, 034101 (2010).

[19] W. B. Zeleny, Phys. Rev. 158, 1223 (1967).

[20] H. Umezawa, Quantum Field Theory (North-Holland, Amsterdam, 1956).

[21] L. M. Abreu, E. S. Santos, and J. D. M. Vianna, J. Phys. A: Math. Theor. 43, 495402 (2010).

[22] L. B. Castro and A. S. de Castro, Phys. Lett. A 375, 2596 (2011). 